\title{
Big Data Value Engineering for Business Model Innovation
}

\author{
Hong-Mei Chen \\ U of Hawaii at Manoa \\ Honolulu, USA \\ hmchen@hawaii.edu
}

\author{
Rick Kazman \\ $U$ of Hawaii at Manoa \\ \& SEI/CMU \\ Pittsburgh, USA \\ kazman@hawaii.edu
}

\author{
Juan Garbajosa \\ Technical University of \\ Madrid (UPM) \\ Madrid, Spain \\ jgs@eui.upm.es
}

\author{
Eloy Gonzalez \\ Indra Software Labs, Spain \\ Madrid, Spain \\ egonzalezort@indra.es
}

\begin{abstract}
Big data value engineering for business model innovation requires a drastically different approach as compared with methods for engineering value under existing business models. Taking a Design Science approach, we conducted an exploratory study to formulate the requirements for a method to aid in engineering value via innovation. We then developed a method, called Eco-ARCH (EcoARCHitecture) for value discovery. This method is tightly integrated with the BDD (Big Data Design) method for value realization, to form a big data value engineering methodology for addressing these requirements. The Eco-ARCH approach is most suitable for the big data context where system boundaries are fluid, requirements are ill-defined, many stakeholders are unknown, design goals are not provided, no central architecture pre-exists, system behavior is non-deterministic and continuously evolving, and co-creation with consumers and prosumers is essential to achieving innovation goals. The method was empirically validated in collaboration with an IT service company in the Electric Power industry.
\end{abstract}

\section{Introduction}

Big data represents unprecedented opportunities for enterprises to compete on analytics [5]. Much value is expected to be derived from high velocity, massive volumes of data from everywhere, including operation optimization, customer intelligence and product/service innovation. Many enterprises are hoping to capitalize on big data for "game-changing" innovations that could fundamentally transform organizational processes, business models and strategies, and even entire industries and markets [13][15][18].

However, how to engineer value from big data poses many new challenges. (To engineer means that a set of procedures can be applied with predicable results [6][9].) Organizations face challenges due to: (1) the technical complexity arising from the $4 \mathrm{~V}$ (Volume, Variety, Velocity, Veracity) characteristics of big data [16]; (2) the organizational agility required for rapid delivery of value [5]; and (3) the rapid technology proliferation and evolution [12].

Value engineering has traditionally focused on improving the "value" of goods or products and services by focusing on functionality. Value was defined as the ratio of function to cost. For more than a decade, value-based software engineering [3] has called for attention to business value and not just software development costs and schedule issues. It advocates integrating value considerations (in contrast to being value-neutral, in which every requirement, use case, object, test case, and defect is equally important) into software engineering principles and practices. Value-based requirements analysis or value analysis involves an approach to improving the value of an item or process by understanding its constituent components and their associated costs. Value-based architecture analysis methods have also been proposed, such as the CBAM [24], which considers the cost, benefits and schedule implications of different architectural strategies.

Similarly, economics-driven software engineering is a stream of research that focuses on value, for instance, the ROI of techniques such as refactoring, and technical debt management. Service engineering [6][9] is another research area that has made the issue of business value salient and also integrates corporate governance measures to improve the costeffectiveness of business-IT alignment. Service engineering/science distinguishes two types of value: static "value in exchange" and dynamic "value-inuse". Value-in-use is co-created with customers and partners in an eco-system [7][8]. The focus of both service engineering and economics-driven software engineering is on a conscious and explicit set of disciplined procedures designed to seek out optimal values for both initial and long-term investment.

Big data value engineering shares the same goals. However, through an exploratory multiple case study [15], we found existing methods for "small data" systems are inadequate for the big data context where data sources, system functions, requirements and hence "value(s)" are continually moving targets. In addition, risks in the big data world are substantially greater, given the large amount of up-front 
investment and the rapidly changing big data technology landscape. More importantly, enterprises' desires for game-changing innovation highlights the need for creativity in addressing the indeterminacy of wicked problems [33] [2] and calls for design thinking that goes beyond traditional "small data" problem-solving paradigm.

Our research aims to address the research question of how to engineer value from big data for gamechanging innovation. Following the Design Science research approach [23], first, employing an exploratory multiple case study and literature review, we identified the problems, defined the objectives of solutions and formulated the requirements for big data value engineering for innovation. Second, employing a collaborative practice research (CPR) [16] approach, we developed a big data value engineering methodology, which include multiple methods for the full lifecycle of big data value engineering. Third, we validated the methods using case studies.

In this paper, we will focus on an empirical case study with our case company (which we refer to as IND) in the Electric Power industry, which has been experiencing a sea change. The case was appropriate for validating our method because no central architecture pre-exists, system behavior is nondeterministic and continuously evolving, and cocreation with consumers and prosumers is essential to achieving innovation goals. We will describe how IND failed in their initial attempts at envisioning and designing a big data system, and then applied our method to discover value from big data and generate a new business model. We called this new business model "eBay in the Grid" where utilities are not just suppliers of power for traditional consumers but also platform providers, on analogy with eBay in the eCommence world, for emerging, evolving energy markets. ${ }^{1}$

\section{Formulating Requirements for a Big Data Value Engineering Method}

To understand the requirements for an effective big data value engineering method, we conducted an empirical multiple case study of 25 European enterprises: 23 large enterprises, 1 medium, and 1 small. These 25 companies responded to our email invitations to 60 companies to participate in our

\footnotetext{
${ }^{1}$ An earlier, shorter version of this paper appeared in the $2^{\text {nd }}$ International Workshop on Big Data Software Engineering, Austin, TX, May 2016, entitled "Toward Big Data Value Engineering for Innovation”.
}

research. The average number of employees of our case enterprises was greater than 150,000. There were 5 outsourcers and 20 non-outsourcer large enterprises. 21 were German-based, with another 4 in Europe but outside Germany. 1 of the 4 non-German companies was headquartered in the U.S. The case companies are in a wide variety of industries. These include: telecommunications; automation \& power; airplanes; global financial services; logistics; airline; reinsurance and financial services; smart plants; conglomerate; financial services; automotive components; automobile manufacturing; investment banking; energy utility, telecom, and IT; insurance software; tax and legal software; general outsourcing; energy technology outsourcing; smart city technologies: telecom IT services. We collected data from multiple sources including public corporate information, management consultant reports, magazine and newspaper articles, informal exchanges, formal interviews, site visits, documents (presentations, internal reports, use cases, etc.) provided by the case companies, and collaborative practice research (CPR) with 2 outsourcers. One author worked for one of the conglomerates. We conducted 28 formal semi-structured interviews with 40 individuals, each of which lasted 1 to 3 hours.

Our exploratory research findings showed that big data deployment is scarce and many enterprises are struggling to deal with the complexity associated with the system development paradigm shifts [15][16] - existing methods are inadequate. Our result also revealed that big data adoption shifts the focus from the technology to the business model and from problem-solving to innovation, departing from previous IT adoption trends. Different from previous IT adoption, most enterprises desire game-changing innovations from big data, not just incremental improvements of existing business models.

Our research also showed that a new process called "Value Discovery" has become common for big data system development in practice. This is because big data adoption is surrounded by high levels of risk and uncertainty regarding costs, schedules, and benefits. Before deployment, our case companies would engage in a top-down innovation process-for some companies this process alone would take 3 or more years - to discover value from big data. Such a value discovery process is unique and critical for big data engineering. A full life cycle of big data value engineering would include value discovery and realization phases with activities to implement the realization of discovered value. However, applying traditional "small" data system design thinking and system development approaches to big data value discovery and realization is 
problematic and inadequate to meet the following requirements (REQs) uncovered in our search for an effective big data value engineering method:

\section{REQ-1: Design thinking for Innovation:} Enterprises, first and foremost, desire innovation from big data: they seek transformational business opportunities and new business models. They want to be the "Uber" of their industry. The types of value expected from big data are a departure from the conventional "problem solving" paradigm in software and system engineering where requirements can be well-defined. In our exploratory multiple case study, some organizations describe big data as "hammers looking for nails," coming from a problem-solving paradigm and thus they were unsuccessful in adopting big data. Those who did adopt big data did not see big data as a hammer. The focus in these successful cases was on design intention (mindfulness) for innovation and called for a design attitude and method for dealing with the constant and yet continually unexpected, possibly disruptive, innovations in the big data technology ecosystem.

REQ-2: Design for the Open World: The large variety of data from everywhere is an enormous opportunity for a big data system. It offers opportunities for forming alliances and collaborating with different partners in the supply network and for co-creating value with customers and prosumers [26]. What data sources to include is, however, not a static requirements problem. Traditional value engineering and small system development methods were largely based on closed world assumptions, which analyzed the requirements of a project for the purpose of achieving the essential functions at the lowest total costs over the life of the project. The closed-world perspective also assumes that an enterprise has control over the systems designed and that design outcomes are largely deterministic. The fluid system boundaries in the big data world challenges the old paradigm and requires a new design approach.

REQ-3: Integrating Value Discovery with Value Realization: Big data value discovery is inherently a creative effort. There exist many ideation techniques, such as brainstorming (perhaps with electronic support such as Group Decision Support System), technology roadmapping, "blue ocean" strategies [28], etc. which were created to help develop scenarios or business cases. However, these techniques alone fall short for big data value engineering as the scenarios created are not conducive for value realization, e.g., requirements negotiation, architecture design and subsequent system development activities. Existing ideation methods offer little in the way of systematic assistance in reasoning about the risks, costs, and benefits associated with scenarios.

Studies have also shown that "separation of concerns" in traditional software engineering is not conducive for value creation [3][24]. For instance, architecture design and requirements negotiations are conceptually tightly related but often performed separately in real-world software development projects. As our prior case studies (e.g. [25]) have revealed, this separation can cause uncertainty in requirements negotiation that hinders progress, limits the success of architecture design, and leads to wasted effort and substantial re-work later in the development life-cycle. It is particularly important for big data value engineering that a method can assist stakeholders to create new scenarios, and to elicit, explore, evaluate, negotiate, and agree upon architecture alternatives based on their understanding of the implications of each scenario. Such an integration will create a 'generate-and-test' process in rapid iterations. The stakeholders can better understand the ramifications of their requirements (expressed as scenarios) in terms of their conflicts with other requirements, their costs, their schedule implications, and their benefits along multiple quality attribute dimensions. As such, the stakeholders can make better decisions about their requirements and prioritize scenarios based on better informed and more holistic value decisions.

REQ-4: Support for Value Experimentation and Verification: Value engineering elicits ideas on alternative ways of maintaining or enhancing results while reducing life cycle costs. Value engineering can be applied at any point in a project, even in construction. However, typically the earlier it is applied the higher the return on the time and effort invested. Due to the scale and scope of big data projects, estimating total cost of ownership is difficult and complex and the system qualities (performance, scalability, interoperability, availability, etc.) cannot be cost-effectively measured by traditional horizontal prototyping methods. The rapid rate of technology proliferation and evolution in the big data area also creates problem for value assessment. We have thus developed an architecturecentric approach, combined with strategic prototyping, to address this concern [12].

\section{An Eco-Architecture Approach}

To meet the above-mentioned requirements, this paper argues for an eco-architecture approach for big data value engineering under high uncertainty. The Eco-ARCH method was originally developed for 
ultra-large systems where no architecture exists [10][27]. An exploratory action research was initially conducted on the Demand-Response (DR) component of the U.S. Smart Grid for design, development and validation of the Eco-ARCH method. The Smart Grid is a complex multi-layered ecosystem composed of an enormous number of constituent systems; every electric utility company employs many constituent systems. There is no central planning for the system architecture although there are some constraints, such as policies, contractual agreements, and legal requirements addressing issues of continuity of service and public safety. Electric utilities are being asked to plan for the Smart Grid of the future where technologies and consumer behavior are constantly changing.

The steps of the Eco-ARCH method are illustrated in Figure 1. Each step of ECO-ARCH contains two levels of analysis: Macroscopic and Microscopic. Note that there are iterations between levels and among the steps. The method provides paired macroscopic and microscopic analyses, supporting innovation in dealing with wicked problems while offering a rational design process based on proven engineering techniques for meeting quality attribute requirements and integrating with "triple bottom line" goals: profit, people, and planet. It advances the frontiers of design science to deal with indeterminacy in system requirements, system behaviors and design outcomes. It also advances architecture analysis by focusing on the architecture landscape, which encompasses all architecture choices and the possible alternatives for the creation and operation of the system, in an open ecosystem, instead of the single, concrete architecture assumed in traditional methods.

We integrated the Eco-ARCH method as the basis for big data value engineering because it embodies design thinking rooted in expandable rationality (addressing design for innovation) [21][22] and employs rigorous engineering principles (addressing efficient problem-solving). This method encourages a "futuring" mindset via ecosystem-wide scenario brainstorming, guides the construction of an architecture landscape for risk analysis, and uses balance-scorecard techniques for cost-benefit analysis. Value-based requirements analysis is integrated with the first step of value realization, as we will show (in Figure 2).

We must here distinguish between "design" (for example, making a graphic cover for an album, or composing a song) and "problem solving" (e.g., playing chess, doing a puzzle). Design is essential to innovation and problem-solving is a moment in design. Engineering is fundamentally a problem solving paradigm grounded in "bounded rationality" [35] - essentially a search through a space of possible solutions, ending when a satisficing (i.e., good enough) solution is found. Since the bounded rationality paradigm treats design as a problemsolving activity it may limit creativity. Design thinking based on "expandable rationality" [21][22] sees problem-solving as only a moment within a design process.

Eco-ARCH breaks from the old engineering paradigm. The basic premises of expandable rationality are that: 1) design problems are wicked problems, 2) design problems are not fully knowable and they evolve during the process, 3) a design attitude sees problems as opportunities for the invention of new alternatives, and 4) problem solving is a subset of innovative design.

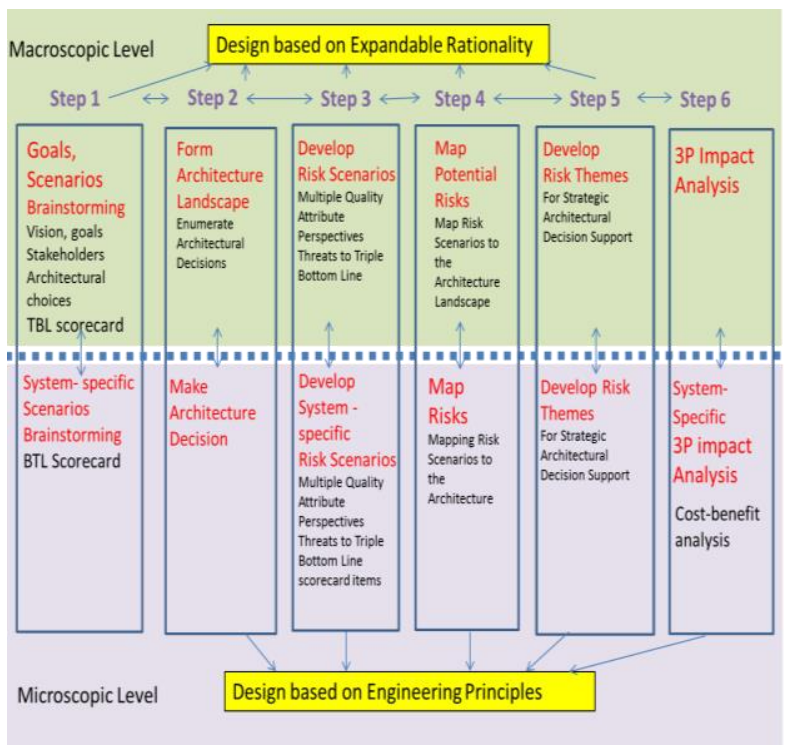

Figure 1: The Original Eco-ARCH method [10]

\section{Integrating Eco-ARCH Method into the Big Data Value Engineering Methodology}

Value engineering includes two phases: Value Discovery, and Value Realization. We have augmented the original Eco-ARCH method [10] for big data value discovery with: 1) "Priming" techniques [19] for futuring scenario generation, 2) a Big Data Architecture Scenario (BDAS) template for big data modeling, 3) a Big Data-Data Flow Diagram (BD-DFD) for process modeling, and 4) strategic prototyping [12] to meet the requirements stated above. These augmentation techniques were each 
validated independently before being integrating into Eco-ARCH.

The augmented Eco-ARCH method is integrated into the Big Data Design (BDD) methodology [16][14] which focuses the practitioner on value realization. The steps of the augmented method are depicted in Figure 2. Steps 0 to 7 are in the Big Data Value Discovery phase while Steps 8-10 are in the Big Data Value Realization phase. We will only briefly describe these steps as BDD has been validated in separate studies and described elsewhere in detail (in [16] [5]). Included in the augmented EcoARCH Steps 4-7 are steps for integrating value discovery and value realization as REQ-3 stipulates. REQ-4 is met specifically in Steps 6-7 that support value experimentation and validation. REQ-1 and REQ-2 are satisfied by Steps 1-4.

There is an important distinction in using the method for 1) business model innovation and 2) for improvement within an existing business model. For business model innovation, an architecture landscape is used (as shown in Step 4 in Figure 2). For improvement under existing business models, it may be sufficient to use reference architectures (not architecture landscape) as the starting point for design as described in [5][14].

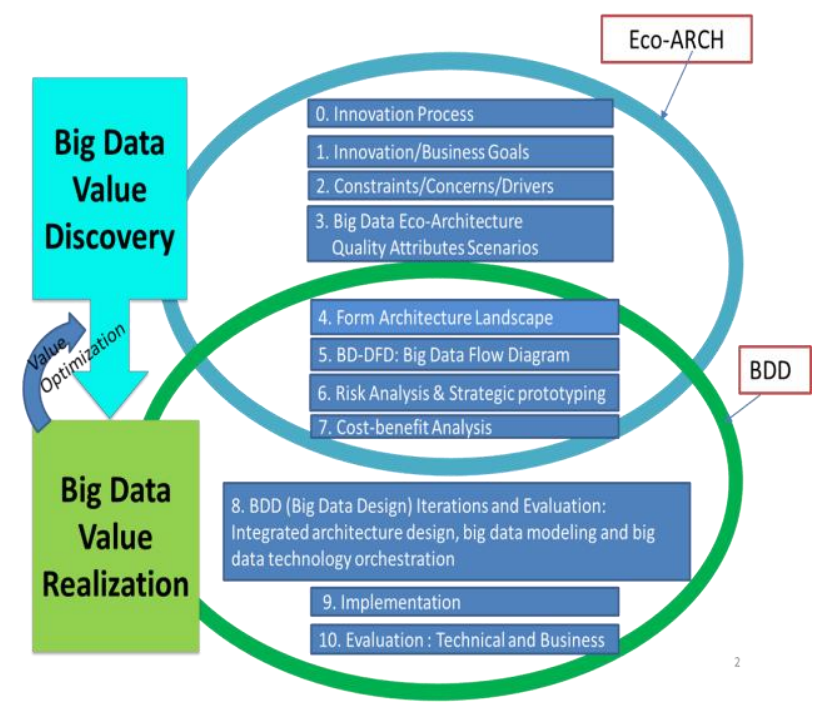

Figure 2: The Eco-ARCH method integrated into the Big Data Value Engineering Methodology

Step 0 - Innovation Process: A top-down innovation process is essential to involve as many stakeholders as possible [15][13]. In many cases, stakeholders are not known at the beginning and the set of stakeholders will evolve and new ones will be included when appropriate.
Step 1 - Innovation/business Goals: Eco-ARCH starts with brainstorming for defining business and innovation goals. It's important to embrace "mindfulness" for innovation to discover value. An organization must explicitly attend to innovation for it to occur; this is known as innovation mindfulness [20]. The architecture or system design is an important tool to effect innovation based on this mindfulness perspective. It employs a futuring technique: stakeholders are guided to free themselves from existing conditions and existing business models and imagine what the future would be. At every brainstorming workshop, participants are "primed" to envision future systems for big data. Following a value-based approach, different goals will be voted on and prioritized. The top 5 goals (or any smaller number of goals agreed upon by the stakeholders) will be selected for expressing as quality attributes scenario in Step 3.

Step 2 - Constraints, Concerns, and Drivers: Unlike the traditional single-architecture system, the constraints, concerns and drivers [4] for the big data ecosystem are brainstormed and then modeled, rather than being based on existing business models. Risks and costs are often viewed as drivers in architecture decision-making and hence Eco-ARCH has incorporated approaches such as the Cost Benefit Analysis Method (CBAM) [24] that consider architectural decisions as investment decisions, as shown in Step 7.

Step 3 - Big Data Quality Attribute Scenarios: In this step, new scenarios are brainstormed for each prioritized business or innovation goal. Ideas for innovation are elaborated and modeled using big data quality attribute scenarios [1]. Each of these scenarios may have an impact on the architectural design decisions that have been made (perhaps even as constraints), or not made. The big data scenarios focus on architecturally significant requirementsquality attributes such as performance, availability, etc. We then record big data modeling inputs using the BDAS template for each scenario. This template captures 14 data architecture elements, including data source quality, data variety, data volume, velocity, $\mathrm{read} /$ write frequency, time to live, queries, OLTP or OLAP, etc. Each input has a direct implication on subsequent architecture choices, data model selection, technology selection, and data access patterns. The template allows easy documentation of the data sources and requirements and facilitates data modeling during Step 8 in the Value Realization phase.

Step 4 - Form Architecture Landscape: An architecture landscape, encompassing all architecture choices derived from the scenarios, is a critical step 
for future systems where no existing system architecture is in place. Each scenario will exercise some architectural alternatives. By considering the full set of scenarios, an architectural landscape may be drawn, showing the possible alternatives for the creation and operation of the system. Each alternative represents a significant architectural decision that must be made. The alternatives may be based upon logical options (e.g. push versus pull communications; acknowledgement of messages or not), commercially available components (e.g. available types of networks or devices), or decisions within an architectural element (e.g. frequency of messaging; message re-send policy).

Step 5 - BD-DFD (Big Data - Data Flow Diagram): Creating the BD-DFD is also a creative process that connects all the data flows and processes in each scenario and composes the context diagram for the architecture vision for the future. The BDDFD can also be generated for a specific instance of architecture by selecting elements in the architecture landscape. Data flow diagrams are a familiar modeling tool, and we did not need to add any new constructs to them. The BD-DFD facilitates big data modeling, in Step 8.

Step 6 - Risk Analysis and Strategic Prototyping: In this step, risks are analyzed using a combination of architecture analysis and strategic prototyping [12] to achieve the value-based objectives. Using architecture analysis, risk scenarios are developed to describe challenges to the system from multiple quality attribute perspectives and threats to the triple bottom line. It is not enough that an architecture works well under normal conditions (those described in the scenarios), but it must work well when stressed, when faced with unexpected demands or failures, or when faced with evolutionary pressures. Risk scenarios are chosen to understand the implications of such challenges on architectural decisions. When risk scenarios are mapped onto an architectural landscape, the assumptions lurking behind each architectural decision become evident. Some of these assumptions, alone or in combination, may pose risks for the achievement of a system's quality attribute goals.

This mapping, along with a model of each quality attribute, is the basis for the architecture analysis in the traditional ATAM [1]. In addition, the risks that we find as a result of the scenario mapping process can be consolidated into risk themes. In mapping substantial numbers of scenarios, we often see the same kinds of risks emerging over and over. Such themes need to be explicitly identified as these pose the greatest risks to the success of the system. An architectural analysis exercise always locates many potential risks but not all risks are equally likely and not all of them have the same set of consequences.

The commonalities in the risks found have led us to "roll up" many of the risks into themes so that these may be made the focus of future investigations.

Architecture analysis is a relatively low cost option for risk analysis. However, there are situations where architecture analysis alone can not provide an adequate understanding of the risks. In such situations, prototyping is often required. In cases where prototyping is involved, we have developed strategic prototyping guidelines [12] for creating throwaway or vertical prototypes, rather than more expensive horizontal prototyping. Note that strategic prototyping in value discovery phase is different from "small" data system development where horizontal prototyping is the norm and it is often conducted in the value realization phase.

Step 7 - Cost-benefit Analysis: In this step, we employ techniques such as the CBAM [24] to do a cost-benefit analysis of the architecture decisions and risks uncovered with respect to the business and innovation goals. Risks are potential problems, and we strive for early identification of risks as a means of assessing their impact and preventing them from being realized. Each risk might then be further analyzed in more detail, e.g., by building a performance model, or by creating a simulation, an experiment, or a prototype. And each of the architectural decisions that go into this risk should be keenly scrutinized by any architect, at both macroscopic and microscopic levels.

Step 8 - Big Data Design (BDD): In this step, an architect must choose specific architecture elements-patterns and technologies-from the architecture landscape to form an implementable instance of a system. The details of the BDD steps are described in [16][14] [5] and depicted in Figure 3. BDD tailors and augments ADD (Attribute-Driven Design) [4] for combining architecture design, big data modeling and technology selection in an iterative design process to optimize each iteration goal.

\begin{tabular}{|c|c|c|c|}
\hline System Architecture & \multicolumn{2}{|c|}{ Big Data Modeling } & $\begin{array}{l}\text { Technology } \\
\text { Selection }\end{array}$ \\
\hline 1. Choose Reference & itecture & \multirow{2}{*}{ 3. BD-DFD } & \\
\hline \multicolumn{2}{|c|}{ 2. Architecture Landscape Instance } & & \\
\hline \multicolumn{3}{|c|}{ 4. Establish Iteration Goal } & Design Concepts \\
\hline \multicolumn{3}{|c|}{ 5. Choose Element(s) to Decompose } & Catalog \\
\hline \multicolumn{3}{|c|}{ 6. Choose Design Concepts + Data Models } & \\
\hline \multicolumn{3}{|c|}{ 7. Instantiate Architectural Elements } & \\
\hline \multicolumn{3}{|c|}{ 8. Sketch Views \& Record Decisions, Metadata } & Big data \\
\hline \multicolumn{3}{|c|}{$\begin{array}{l}\text { 9. Evaluation of Each Iteration } \\
\text { (may include prototyping, scale-up testing) }\end{array}$} & Catalog \\
\hline \multicolumn{3}{|c|}{ 10. Architecture Analysis and Evaluation: BITAM } & \\
\hline
\end{tabular}




\section{Figure 3: Design steps of the BDD method}

\section{Empirical Case Study}

To validate the Eco-ARCH method, our criteria for case selection include the following system context:

1. system boundaries are fluid,

2. requirements are ill-defined,

3. many stakeholders are unknown,

4. design goals are not provided,

5. no architecture pre-exists,

6. system behavior is non-deterministic and continuously evolving, and

7. co-creation with partners, consumers, and prosumers is essential to achieving innovation goals.

Our case company, IND, is in the electric power industry, which meets all our criteria. Today there is a sea change occurring in the electric power industry. The disruptive developments in smart grid distributed energy generation and distribution, the rapid growth and viability of renewable energies, the rise of "energy communities", the proliferation of demand response-enabled smart devices and the threat from new battery technologies (e.g., graphene) all contribute to force utilities out of their "natural monopoly" status. Existing utility control systems are not able to manage the physical infrastructure being added to the grid (e.g., solar panels, wind turbines, customer-owned microgrids, smart devices, etc.), let alone dictate real-time market exchanges. To survive, the utilities must change their business models and rethink their role in the value proposition.

IND has 44,000 employees providing outsourcing and integration services. Our case study was performed with the energy R\&D Division from Fall 2014 to Summer 2015, generating a new business model in value discovery, and then continued into 2016. The R\&D division focuses on two areas: 1) distribution operation and automation and 2) network monitoring and control operations: real time and quasi-real-time information for managing networked smart devices for the electrical distribution application domain. Smart grid management requires dealing with huge amounts of data collected from smart meters and other devices connected to the power network. Currently, data is gathered in large volumes from meters and analyzed "off-line" in timeconstrained periods (quarterly, hourly, daily, and monthly). However, electricity companies are demanding IT solutions to deal with the smart monitoring of power networks and perform big data analytics to drive insights.
Big data in the existing business model is leveraged for: 1) energy efficiency by analyzing customer consumption summaries or real-time usage, 2) theft detection, 3) load forecasting to optimize utility companies' purchasing and generation decisions, 4) grid utilization, as well as outage prediction and detection, and 5) customer experience. Many use cases have been developed. This domain has many constraints in terms of government regulations and hundreds of standards for smart devices. In addition, there are many suppliers of hardware, software, and services; the market is very granular, with stringent requirements including decision-making in nanoseconds with significant consequences. Facing the current turbulence and uncertainty of the Electric Power industry, IND's strategic focus is to use big data (and IoT) for innovations and new business opportunities.

IND prides itself in innovation. "We use whatever technology is available at the time" stated the division head. They considered big data technology in 2009, but this technology was deemed too immature back then. They experimented with many different combinations of big data technologies and related architectures. The results were, however, unsatisfactory. They had issues with inconsistency of data (for instance, readings could be affected by the weather, by differences in smart devices, etc.) and so they needed to perform extensive consistency checks when they received the data. The data sources were also very diverse and IND was unable to achieve system scalability. They ceased experimentation with big data in 2011 and restarted in 2014. They have experimented with edge computing and fog computing for putting control and data processing power into smart devices to speed up data retrieval time.

Pursuing innovation, IND agreed to follow the Eco-ARCH method for big data value discovery. Two of the authors of this paper were creators of the Eco-ARCH method and were facilitating the steps for IND. Their high-level innovation goals, after voting and prioritization, were:

1. Reduce cost

2. Increase capabilities/quality (attributes)

3. Improve market position: product lines, time to market, differentiating features

4. Improve business processes: better, faster, smarter, cheaper, employee training/retention, DevOps

5. Improve confidence and image of the system: end users, customers (utilities), partners

All 5 of these goals are, in the end, related to Goal 2 -without increasing their underlying capabilities, none of the other goals would be possible. Given 
these goals the IND stakeholders proceeded to generate big data quality attribute scenarios: 17 stakeholders generated over 50 scenarios. As a way to classify them and understand whether they had achieved adequate coverage, the scenarios were mapped to the current TC57 reference architecture, as shown in Figure 4.

After the big data scenarios were mapped and the BD-DFD was sketched, a new business model emerged, which we called "eBay in the Grid". The idea was that, in the past, utilities and service providers saw themselves as primarily creators of systems that had relatively limited objectives: generation, transmission, distribution, accounting, and so forth. By considering the architecture landscape, IND saw a new business opportunity in building a platform, providing a broad basis of generic services, data, and analytics upon which others could build value-added services.

Value co-creation scenarios were further brainstormed to solidify the detail of the new business model, as shown in Figure 5 .

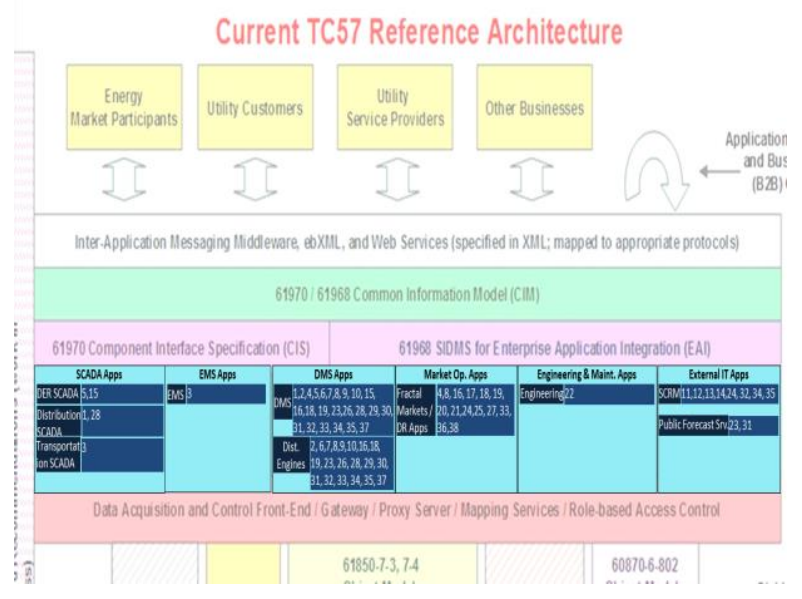

Figure 4: The TC57 reference architecture annotated with brainstormed scenarios

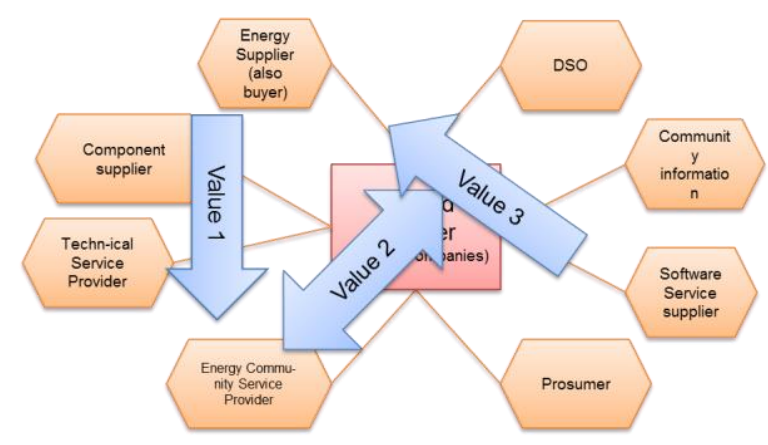

Figure 5. Value based on agents' perspective
The new business model moved them from being an electricity supplier (a goods-dominant perspective) to a smart service provider in the future Transactive Energy ecosystem (a service-dominant perspective), including:

1. Enabling B2C businesses for energy related services of all kinds,

2. Creating a network of suppliers and buyers for energy related equipment and components,

3. Bringing advanced energy services to energy communities, and

4. Connecting all energy markets and energy community stakeholders for timely, trusted and correct information on energy delivery and security of supply.

The feedback that we received from IND in using the method was as follows:

1. The method allows them to think beyond their current state and guide to systematically explore various innovation options that they had not conceived before.

2. It was challenging for them to think beyond the current state at the beginning but after several working sessions, they were able to formulate quality attribute scenarios much more easily.

3. It was also difficult for them to understand what requirements were "architecturally significant" and what are not.

4. The priming exercises were received positively. They said that it cleared their minds and aided in the brainstorming process.

5. The discovery of a new business model was a "nice surprise".

\section{Discussion}

Issues and limitation are worth noting:

First, a complete and authoritative validation of Eco-ARCH is impossible. The concept of an architectural landscape, along with techniques for envisioning, analyzing, and scoring realizations of the landscape is, we believe, our most important contribution. A key element of Eco-ARCH is expansive design thinking for innovation, and the outcomes from this process-the architecture landscape and associated scenarios and risks. The discovery of new kinds of value propositions, such as a new business model in our case, are obviously useful but it must be noted that the exact conditions to induce such creativity are hard to pinpoint and replicate. 
Second, Eco-ARCH guides the stakeholders to analyze potential risks lurking within the landscape, their consequences, their interactions, and their tradeoffs. Eco-ARCH supports risk-based reasoning for cost-benefit analysis of architecture decisions, which are investment decisions.

To validate the effectiveness of the method, we examined two aspects of the risks discovered: coverage and correctness.

1. Did we find a majority of the most important risks?

2. Were the risks that we found truly significant challenges to the achievement of some important system goal?

When performing an architectural evaluation using a technique such as the ATAM, correctness and coverage are reasonably easy to achieve, as long as the method is faithfully prosecuted.

When evaluating an underspecified big data architecture with multiple unknown and unknowable stakeholders, it is not possible to get agreement on the risks. Hence we must rely on the stakeholders present as proxies. There is another, more fundamental issue with all risk evaluation and prevention methods. How do you measure the benefit of the risk avoided?

Third, a limitation of the present study is regarding the generalization of the case method. Thus far we have only worked with a single organization, although many parts of Eco-ARCH have been validated independently through other case studies.

Fourth, Eco-ARCH, like all methods cannot guarantee that the resulting system will be usable or that the policies that these systems enact will be attractive to all involved. For successful big data value engineering, the system must be easy to change such policies, with few ripple effects on other parts of the system.

Fifth, The Eco-ARCH method relies heavily on brainstorming for scenarios. Although scenarios have been heavily used in architecture design and analysis in the past, it cannot be overstated that the quality of the scenarios generated is critical. Expandable rationality design thinking focuses on a "breadthfirst" strategy to co-evolve both problems and solutions, which may generate large numbers of scenarios. This is where our value-based approach comes in where scenarios were voted and prioritized (based on negotiation among stakeholders). However, this will depend on the facilitators' skills in guiding and managing the process.

Sixth, the successful use of Eco-ARCH will rely on a top-down innovation process, requiring an alignment with an organizational culture that fosters innovation, is open to change, and possesses (or is willing to construct) an agile infrastructure. Eco$\mathrm{ARCH}$ can assist big data value discovery and facilitate big data value realization with architecture agility, but the organizations must have innovation "mindfulness" to achieve innovations goals.

Seventh, the successful use of Eco-ARCH also depends on the ability of the architects. Traditional IS and CS curricula emphasize engineering approaches to problem-solving. As a result, it is difficult for software engineers and architects to make the transition to a more open, creative space. We will have to rethink IS curricula to address this challenge for big data value engineering.

\section{Conclusions}

Big data value engineering for business model innovation requires new methods. Eco-ARCH embodies design thinking rooted in expandable rationality ideal for big data discovery. It provides a dual macroscopic-microscopic analysis technique, allowing for innovation in dealing with wicked problems in the big data open world while offering solid engineering-based design with proven techniques for system-specific quality attribute evaluation, risk-based cost-benefits evaluation. We augmented Eco-ARCH with four additional contributions: 1) "Priming" techniques for Futuring scenario generation, 2) a Big Data Architecture Scenario (BDAS) template for big data modeling, 3) a Big Data-Data Flow Diagram (BD-DFD) for process modeling, and 4) strategic prototyping.

Eco-Arch contribution of the architecture landscape addresses open world design problems while big data quality attributes scenarios facilitate data modeling in subsequent design steps. Strategic prototyping is an integral part of Eco-ARCH, utilizing the value-based engineering principle. As such, Eco-ARCH meets all the requirements (e.g., REQs 1-4 formulated in our multiple-case study) for a big data value engineering method. Employing the Eco-ARCH method, our case company was able to reframe their value proposition, transitioning from goods-dominant logic to service dominant logic. They were able to conceive a radically new business model for tackling business transformation imperatives in the Electric Power industry and achieve their innovation goals.

\section{References}

[1] Bass, L., Clements, P., and Kazman, R. 2012. Software Architecture in Practice, 3rd ed., Addison-Wesley.

[2] Buchanan, R. 1992. "Wicked problems in design thinking”, Design Issues (8:2), pp. 5-21. 
[3] Boehm, B. "Value-Based Software Engineering: Overview and Agenda", USC Technical Report, USCCSE-2005-504.

[4] H. Cervantes, R. Kazman, Designing Software Architectures: A Practical Approach, Addison-Wesley, 2016.

[5] Chen, H-M, Kazman, R., and Haziyev, S. "Agile Big Data Analytics for Web-based Systems: An Architecture-centric Approach", IEEE Transactions on Big Data, in press, June 2016

[6] Chen, H-M. 2008. "Towards Service Engineering: Service Orientation and Business-IT Alignment", Proceedings of the Hawaii International Conference on System Science (HICSS-41).

[7] Chen, H-M., and Vargo, S. "Service-Oriented Challenges for Design Science: Charting the "E"-volution", Pacific Asian Journal of the Association of Information Systems (PAJAIS), (2:1), March 2010, pp.1-15.

[8] Chen, H-M., and Vargo, S., 2008. "Toward an Alternate Logic for Electronic Customer Relationship Management", International Journal of Business Environment (2:2) pp. 116-132.

[9] Chen, H-M, Kazman, R., and Perry, O. "From Software Architecture Analysis to Service Engineering: An Empirical Study of Methodology Development for Enterprise SOA Implementation", IEEE Transactions on Services Computing, April-June 2010 (vol. 3 no. 2), pp. 145-160

[10] Chen, H-M, and Kazman, R. 2012. "Architecting for

[11] Ultra Large Scale Green IS", Proceedings of GREENS 2012: First Workshop on Green and Sustainable Software (GREENS) at ICSE 2012.

[12] Chen, H-M, Kazman, R., and Haziyev, S. "Strategic Prototyping for Developing Big Data Systems", IEEE Software, March, 2016.

[13] Chen, H-M, Schütz, R., Kazman, R., and Matthes, F. 2016. "Amazon in the Air: Innovating with Big Data at Lufthansa", Proceedings of the Hawaii International Conference on System Science (HICSS-49).

[14] Chen, H-M, Kazman, R., and Haziyev, S. "Agile Big Data Analytics Development: An Architecture-centric Approach", IEEE Proceedings of the Hawail International Conference on System Science (HICSS-49).

[15] Chen, H-M, Kazman, R., and Matthes, F. "Demystifying Big Data Adoption: Beyond IT Fashion and Relative Advantage", Proceedings of Pre-ICIS (International Conference on Information Systems) DIGIT workshop.

[16] Chen, H-M, Kazman, R., Haziyev, S. and Hrytsay, O. 2015. "Big Data System Development: An Embedded Case Study with a Global Outsourcing Firm", Proceedings of BIGDSE'15 (Big Data Software Engineering) Workshop at ICSE 2015.

[17] Clements, P., Kazman, R., and Klein, M. 2001. Evaluating Software Architectures: Methods and Case Studies, Addison-Wesley.

[18] Davenport, T.H., Barth, P., and Bean, R. 2012. "How Big data is Different”, Harvard Business Review (90: 10), pp. 78-83

[19] Dennis A. R., Minas R. K. and Bhagwatwarb A. P. 2013 "Sparking Creativity: Improving Electronic Brainstorming with Individual Cognitive Priming", Journal of Management Information Systems, Volume 29, Issue 4, 195-216.

[20] Fishman R. G. 2004. "Going Beyond the Dominant Paradigm for Information Technology Innovation
Research: Emerging Concepts and Methods", Journal of the Association for Information Systems (5:8), pp. 314355.

[21] Hatchuel A. 2002. "Towards Design Theory and Expandable Rationality: The unfinished program of Herbert Simon", Journal of Management and Governance (5:3-4), pp. 260-273.

[22] Hatchuel, A., and Weil, B. 2009. "C-K Design Theory: An Advanced Formulation", Research in Engineering Design (19), pp. 181-192.

[23] Hevner, A., March, S., and Park, J. 2004. "Design Science in Information Systems Research", MIS Quarterly (28:1), pp. 75-105.

[24] Kazman R., Asundi J., Klein M. "Quantifying the Costs and Benefits of Architectural Decisions", Proceedings of the 23rd International Conference on Software Engineering (ICSE 23), pp. 297-306.

[25] Kazman, R., In, H. and Chen, H-M. 2005, "From Requirements Negotiation to Software Architecture Decisions". Information \& Software Technology, 47(8), pp. 511-520.

[26] Kazman, R., and Chen, H-M. 2009. "The Metropolis Model: A New Logic for the Development of Crowdsourced Systems", Communications of the ACM (52:7), pp. 76-84.

[27] Kazman, R., Bass, L., Ivers, J., and Moreno, G. 2011. "Architecture Evaluation without an Architecture: Experience with the Smart Grid", Proceedings of 33rd International Conference on Software Engineering (ICSE 33).

[28] Kim, S., In, H., Baik, J., Kazman, R., and Han, K. 2008. "Escaping from Red Ocean with Value-Innovative Requirements", IEEE Software, January/February 2008, pp. 80-87.

[29] Kimbell, L., and Street P.E. 2009. "Beyond design thinking: Design-as-practice and designs-in-practice", CRESC Conference, Manchester.

[30] Naedele, M., Chen, H-M, Kazman, R., Cai, Y., Xiao, L., and Silva, C.V.A. 2015. "Manufacturing Execution Systems: A Vision for Managing Software Development", Journal of Systems and Software, Volume 101, March 2015, pp. 59-68.

[31] Northrop, L., Feiler, P., Gabriel, R., Goodenough, J., Linger, R., Longstaff, T., Kazman, R., Klein, M., Schmidt, D., Sullivan, K., and Wallnau, K. 2006. UltraLarge-Scale Systems: The Software Challenge of the Future. SEI/CMU.

[32] Porter, M., and Kramer, M. 2006. "Strategy and Society", Harvard Business Review (84:12), pp. 78-92

[33] Rittel, H. J., and Webber, M. M. 1984. "Planning Problems Are Wicked Problems", in Developments in Design Methodology, N. Cross (ed.), John Wiley \& Sons, New York.

[34] Sarasvathy S., Dew N., Read S. and Wiltbank R. 2008. "Designing Organizations that Design Environments: Lessons from Entrepreneurial Expertise", Organizational Studies, 29(03), pp. 331-350.

[35] Simon, H. 1996. The Sciences of the Artificial. MIT Press, Cambridge.

[36] Sterman, J. D. 2001. "System Dynamics Modeling: Tools for Learning in a Complex World", California Management Review (43:4), pp. 8-28. 